\title{
Idiopathic recurrent gigantomastia: A case report
}

\author{
Abdenacer Moussaoui ${ }^{1}$, Jaouad Kouach ${ }^{2}$, Amine Ennouhi ${ }^{1}$, Abdellah Babahabib ${ }^{2}$, \\ Youssef Benabdejlil $^{2}$, Driss Moussaoui ${ }^{2}$, Hicham Bakkali ${ }^{3}$, Mohamed Dehayni ${ }^{2}$ \\ ${ }^{1}$ Department of Plastic and Aesthetic Surgery, Military Training Hospital Med V, Rabat, Morocco \\ ${ }^{2}$ Department of Gynecology-obstetrics, Military Training Hospital Med V, Rabat, Morocco \\ ${ }^{3}$ Department of Anesthesiology and Critical Care, Military Training Hospital Med V, Rabat, Morocco
}

\section{Email address:}

kouach_jaouad@yahoo.fr (J. Kouach), babahabib2003@hotmail.com (A. Babahabib), hbakkali@ymail.com (H. Bakkali), Moussaouidriss@Yahoo.fr (RD. Moussaoui), Mohamedehayni@gmail.com (M. Dehayni)

\section{To cite this article:}

Abdenacer Moussaoui, Jaouad Kouach, Amine Ennouhi, Abdellah Babahabib, Youssef Benabdejlil, Driss Moussaoui, Hicham Bakkali, Mohamed Dehayni. Idiopathic Recurrent Gigantomastia: A Case Report. Journal of Surgery. Vol. 2, No. 4, 2014, pp. 54-57. doi: $10.11648 /$ j.js.20140204.11

\begin{abstract}
Gigantomastia is relatively rare and mostly unknown manifestation in its diagnostic and therapeutic approach. It is composed by many categories (idiopathic, Juvenile, pregnancy, Medication) that can affect women with strict profile. We report the case of a very important idiopathic gigantomastia which was operated using a technique with superior pedicle with resection of $5 \mathrm{~kg}$ per breast. The evolution was marked by the occurrence of recurrence at 18 months. Through, the analysis of this observation and review of the literature, the authors review the different aspects of this pathology.
\end{abstract}

Keywords: Gigantomastia, Idiopathic, Hypertrophy, Breast Surgery

\section{Introduction}

Gigantomastia is defined by a breast volume $>1500 \mathrm{cc}$ in response of a hypersensitivity of the breast tissue to estrogen hormones. Juvenile and pregnancy forms are the most common. Idiopathic form is exceptional. It has a specific clinical and pathological definition. Its management involves mostly surgery $[1,2,3]$.

The authors report a new case of idiopathic gigantomastia treated by superior pedicle technique which evolution was marked by a recurrence at 18 months and discuss these aspects through the literature data.

\section{Observation}

Mrs KY... 23 years old, unmarried, nurse's aide, without medical history and no similar cases in her family, was admitted in our service for an important gigantomastia . The beginning was at 21 years-old by a quick increase in breast size. The patient consulted her gynecologist. A hormone balance, including Thyroid Stimulating Hormone (TSH), triiodothyronine (T3), Thyroxine (T4), prolactin, estradiol, testosterone was normal. Brain MRI was also normal. The evolution was marked by the occurrence of spectacular inflammatory episodes disturbing the daily life of the patient.
Progestins injectable where prescribed by her gynecologist (Depoprovera ${ }^{\circledR} 01$ injection / week for three weeks) . A significant regression was observed. Two months later, the course was marked by a rapid re-increase breast volume despite the resumption of progestins injectable.

Physical examination in her admission found a patient weighing $94 \mathrm{~kg}$ for a height of $1.66 \mathrm{~m}$, a body mass index of $34 \mathrm{~kg} / \mathrm{m} 2$.The breast exam noted a very important gigantomastia with nipples that came to the pubic region (Figure $\mathrm{n}^{\circ} 1$ ). Distance nipple-areola complex (NAC) and clavicle plate was $52 \mathrm{~cm}$ on the right side and $50 \mathrm{~cm}$ on the left side.

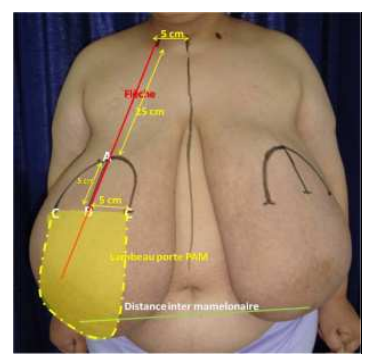

Figure 1. Preoperative appearance. 
Intermamelonnaire distance was $38 \mathrm{~cm}$. On examination, breasts were firm, painless, the skin was thin, distended with exaggeration of subcutaneous venous network .There was maceration in the furrows in breast. The lymph nodes were free.

The ultrasound showed bilateral nodular macro dystrophy. Mammogram showed no abnormalities. After preoperative skin preparation for two weeks with antiseptics until disappearance of maceration, we suggested a breast reduction. Comprehensive information considering the possibility of using the technique Thorek with free graft of nipple-areola complex and possible complications have been provided to the patient .We decided to use a technique of superior pedicle and posterior retaining the possibility of using the graft NAC if a suffering is recognized during the surgery. The procedure is initiated by a patient standing in a upright position, point $\mathrm{A} 25 \mathrm{~cm}$ in Breast axis, the point $\mathrm{B}$ is $5 \mathrm{~cm}$, the width of the future NAC is $12 \mathrm{~cm}$, the length of segment III is $6 \mathrm{~cm}$, a flap door de-epidermization NAC was conducted in the beginning of the surgery. The flap was $12 \mathrm{~cm}$ wide (Figure 2).

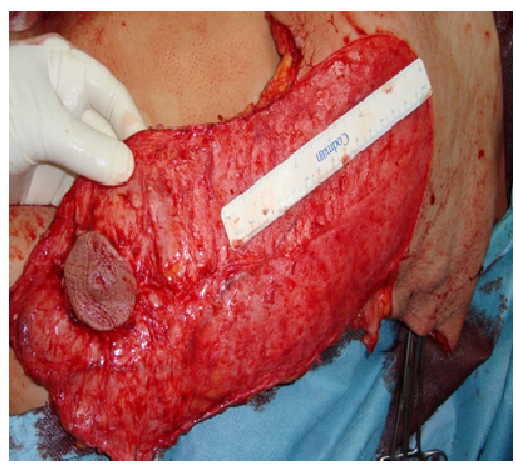

Figure 2. Flap door nipple (upper pedicle)

Excision concerned the lower pole and the external and internal pillars, without detachment of the posterior gland which has kept a post pedicle face. NAC is transposed without difficulty, closing is performed reverse $\mathrm{T}$ (Figure 3a -b). Weight removal : $4800 \mathrm{~g}$ fot the right breast and $4600 \mathrm{~g}$ for the left breast. No intraoperative transfusion was necessary.

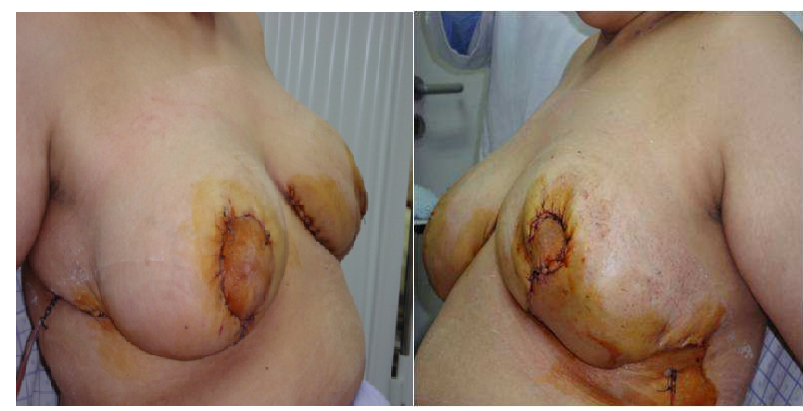

(a)

(b)

Figure 3. Immediate postoperative appearance

The immediate aftermath were simple (Figure 4). The patient was discharged on the 5th postoperative day. Histological analysis showed a dystrophic parenchyma with nodular hyperplasia. One month later, Postoperative recovery was uneventful with a satisfactory appearance of the breast (Figure $\mathrm{n}^{\circ} 4$ ). A Recurrence was observed 18 months after the surgery (Figure $n^{\circ} 5$ ) with a return to baseline at 24 months (Figure $\mathrm{n}^{\circ} 6$ ). Subcutaneous mastectomy with reconstruction aid was offered to the patient but she preferred to abstain.

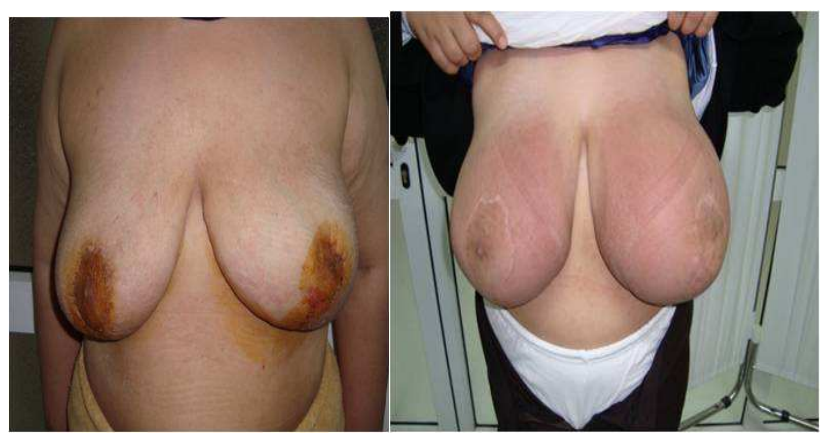

Figure 4. Appearance at 1 month Figure 5. Appearance at 18 months

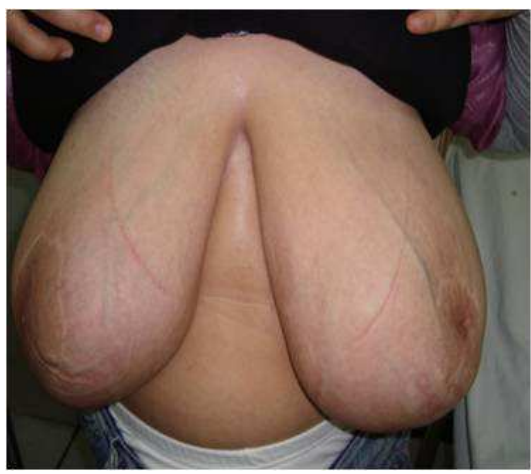

Figure 6. Appearance at 24 months

\section{Discussion}

Gigantomastia is a relatively rare benign condition related to an exaggerated growth of breast volume, usually affecting young woman, responsible of physical and psychological disorders. Durston W is the first in 1969 to describe a case of gigantomastia in the literature [4]. Gigantomastia is defined by a breast volume exceeding $1500 \mathrm{~cm} 3$. Considering that, normal volume varies from 200 to $350 \mathrm{~cm} 3$. Some authors consider it excessive breast growth up to $1500 \mathrm{~g}$ or more $[1,2,3,5]$. Dafydd recently proposed a redefinition of the gigantomastia based on its participation in the body mass index (BMI). We talk about gigantomastia when breast tissue is involved in $3 \%$ or more of the total weight of the patient [2].

In 2008, Dancey proposed a classification into three groups: idiopathic, drug and hormonal stimulation. Gigantomastia related to hormonal stimulation include, the gestational and juvenile gigantomastia. Both types of gigantomastia occurr at different moment in women's life and don't have the same therapeutic indications [1]. It seems 
more logical to separate and define four categories of gigantomastia: idopathique, juvenile, gestational and secondary to medication $[1,4,5,6]$. Gigantomastia of pregnancy is rare. It occurs most often in multiparous women with no particular history at the end of the first trimester of pregnancy. No one factor is retained with certainty $[4,6,7]$. More exceptional is the idiopathic form reaching women over 20 years outside of pregnancy $[1,3,6,7]$. Cases of idiopathic true gigantomastia are close to other subtypes gigantomastia clinical presentation and are rare. Instead, pseudo-gigantomastia in women with a high BMI are the most common gigantomastia. These are gigantomastia which consist largely important to effective series published in recent years. Thus, the mean BMI found in Letertre and Mojjallal series was $32 \mathrm{~kg} / \mathrm{m} 2$ [6,7,8,9]. Each category gigantomastia, although different physiologically, may cause similar symptoms and use the same surgical techniques for the therapeutic component. However, the clinical expression knows some differences and schema support varies [ $3,4,5,6,8]$. The theory of hypersensitivity breast tissue steroid hormones is the most recognized in the pathogenesis of this disease. The pathophysiologic mechanism of occurrence of gigantomastia remains unknown. In idiopathic gigantomasties, when the BMI is high and the installation of the gigantomastia with gradual weight gain, it is probably a related pseudogigantomastie development of adipose tissue. This analysis is confirmed by the histological study which is different from a conventional aspects gigantomastia breast tissue. Therefore propose initial treatment with dietary advice and diet in such gigantomastia seems appropriate before considering surgery $[4,5,7,9]$.

The pathophysiologic mechanism of occurrence of gigantomastia remains unknown. An autoimmune role is mentioned by several authors $[6,9,10]$. In fact, the breast tissue may be affected in some autoimmune diseases. Lupus or inflammatory mastitis diabetics are described. Tournaire studied 8 cases of gigantomastia occurred in patients with an autoimmune disease such as myasthenia, rheumatoid arthritis or thyroiditis [10]. In our case, hormonal assays for FSH, LH, prolactin, TSH, estradiol, testosterone were measured and all proved normal. Radiological evaluation, including mammography, breast ultrasound and MRI revealed no suspicious mass. Histological analysis of the resected breast tissue confirms the diagnosis of gigantomastia and does not highlight formal argument of an autoimmune involvement. However, indirect immunofluorescence showed the presence of antinuclear antibodies. These were non-specific, but the association in this group of patients with an autoimmune disease with antibodies in proliferating breast tissue and normal complementary balance to orient an autoimmune factor in the gigantomastia. Our case illustrate that the idiopathic form is very rare in the literature ( $<10$ cases). It responds to the anatomical definition of this pathology. The impact of such gigantomastia is very important. In addition to the daily challenges, the occurrence of inflammatory processes complicates the lives of these patients with a high risk of maceration or skin necrosis. Respiratory problems weight of the breasts can occur overnight affecting the quality of their sleep. Thus, it is seen as a true social psychological drama see. Postoperatively, our patient has accepted the result, as nurse's aide she enrolled in nursing school.

The management of this disease is primarily surgical but also psychological and medical. Preparation of the patient and her skin is necessary before any surgical procedure, a weight stabilization is necessary in cases of obesity $[1,3,5,8,9]$. The surgical approach is a reduction mammaplasty. Several techniques are used. Free nipple graft or Thoreck is the gold standard for several authors in this type of pathology. Thoreck criticizes were: the risk of partial or total necrosis of the graft, loss of areal sensitivity, inability to breastfeed postoperatively, the discoloration and the default projection of the NAC $[5,6,10,12,13]$.

We never practiced Thoreck and we prefer the technique of Mc Kissok with double pedicle, we used it in all our patients with a very important hypertrophy and in gigantomastia with resection of up to $2 \mathrm{~kg} /$ breast. The pedicle is dermoglandulaire in lower, superior in dermal skin in top. The disadvantages are: difficulties to transpose the NAC with a tendency to expand and postoperative appearance sometimes depressed the NAC. Alternatives to Thoreck techniques are called lower-posterior pedicle and those with superior pedicle $[3,4,6,9,10,11,12]$.

The inferior pedicle techniques are widely used in the USA in the CMF [13,14]. Some authors use a postero-inferior pedicle for weights above $3000 \mathrm{~g}[13,14,15]$. Chang et al have reported success gigantomastia for up to $5100 \mathrm{~g}$ with a rate of necrosis of the NAC $=1.2 \%$ $[9,14,15,16]$. These authors conclude that the reliability of this technique for all types of breast hypertrophy.

The other alternative to Thoreck is the superior pedicle that remain widely used in mild to moderate hypertrophy with high reliability techniques. Their use in gigantomastia is not widespread. Some authors criticize these techniques because it promote areolar necrosis due to the length of the pedicle and its folding $[9,16]$. It has not prevented others to use even in gigantomastia with weights up to $2,200 \mathrm{~g}$ resection $[9,10,11,14,16]$. They insist on the importance of respecting the length ratio / width must not be $>2$.

Other authors have used the technique superior pedicle in hypertrophy with massive ptosis (distance supraclavicular sternal - nipple $>40 \mathrm{~cm}$ ) and they concluded for the safety of this technique. Our case illustrates the combination of a very important gigantomastia to a massive ptosis[6,7,9]. The application of these techniques to higher gigantomastia pedicle must however observe a number of principles $[6,8]$ :

- A wide circle periareolar in order to absorb some of the excess skin and get a wide flap door nipple.

- A retro release areal pre pectoral only in breast axis.

- A flap door nipple end distally and proximally thickening, allowing correct transposition of the NAC and to avoid interruption of venous return after plication.

The occurrence of recidivism is not uncommon, the 
literature is poor. Hormone therapy postoperatively is controversial. Mastectomy with placement of prostheses may be indicated in some cases as a last resort but it is still very aggressive $[1,3,6,7,9,12,13]$.

\section{Conclusion}

Conservative techniques can treat most of gigantomastia. The free nipple graft or Thoreck could be reserved for failures. The recurrences are a real challenge; their support mainly depends on the expectations of the patient. In all cases good information and careful monitoring is required.

\section{References}

[1] Dancey A, Khan M, Dawson J, Peart F. Gigantomastia a classification and review of the literature. J Plast Reconstr Aesthet Surg 2008; 61:493-502.

[2] Dafydd H, Roehl K.R, Phillips L.G, Dancey A, Peart F, Shokrollahi K. Redefining gigantomastia. J Plast Reconstr Aesthet Surg. 2011; 64, 160-163.

[3] Dem A, Wone H, Faye ME, Dangou JM, Touré P. Bilateral gestational macromastia: case report.. J Gynecol Obstet Biol Reprod. 2009: 38, 254-7.

[4] Durston W. Concerning a very sudden and excessive swelling of a woman's breasts. Phil Trans 1969; 4:1047-1049.

[5] Sweltad MR, Sweltad BB, Rao VK, Gutowski KA. Management of gestational gigantomastia. Plast reconstr Surg 2006; 118: 840-848.

[6] Delliere V. Gigantomastie: Proposition d'une prise en charge diagnostique et thérapeutique à partir de cas et d'une revue de la littérature. Thèse de médecine $\mathrm{N}^{\circ} 135,2012$ Faculté de médecine, Université de Nantes

[7] Chargui R, Houimli S, Damak T Khomsi F, Ben Hasouna J, Gamoudi A, Boussen H, Rahal K. Relapse of gigantomastia after mammoplasty. Report of a case and literature review. Annales de chirurgie. Ann Chir. 2005; 13 : 181-5.
[8] Mojallal A, Moutran M, Shipkov C, Saint-Cyr M, Rohrich RJ, Braye F. Breast reduction in gignatomastia using the posterosuperior pedicle: an alternative technique, based on preservation of the anterior intercostals artery perforators. Plast. Reconstr. Surg. 2010; 125: 32-43.

[9] Letertre P, Lasserre G, Ricbourg B. Large breast hypertrophy and gigantomastia management by postero-inferior pedicle reduction technique. About 20 cases. Ann Chir Plast Esthet. 2009 ; 54, 331-9.

[10] Touraine $\mathrm{P}$, Youssef $\mathrm{N}$, Alyanakian MA, Lechat X, Balleyguier C, Duflos C, Dib A, May A, Carel JC, Laborde K, Sigal-Zafrani B, Goffin V, Eymard B, Boitard C, Brousse N, Kuttenn F. Breast inflammatory gigantomastia in a context of immunemediate diseases. J Clin Endocrinol Metab 2005; 90:5287-5294.

[11] Bardot J, Samson P, Aubert JP, Magalon G. Reduction mammaplasty with free nipple. Apropos of 5 cases. Ann Chir Plast Esthet 1995;40:77-82.

[12] Rohrich R.J, Gosman A.A, Brown S.A, Tonadapu P, Foster B. Current preferences for breast reduction techniques : A survey of board-certified plastic surgeons 2002. Plast Reconstr Surg 2004;114:1724-33.

[13] Georgiade GS, Riefkhol R, Georgiade NG. The inferior dermal pyramidal type breast reduction. Long term evaluation. Ann Plast Surg 1989;23:203-11.

[14] Neuprez A, Haykal S, Calteux N. The use of an inferior dermo-glandular flap in Thorek's technique, based on a series of 21 cases. Ann Chir Plast Esthet 1999;44:231-7

[15] Hawtof DB, Levine M, Kapetansky DI, Pieper D. Complications of reduction mammaplasty : comparison of nipple areolar graft and pedicle. Ann Plast Surg 1994;33:561-4.

[16] Mojallal A, Comparin JP, Voulliaume D, Chichery A, Papalia I, Foyatier JL. Reduction mammaplasty using superior pedicle in macromastia . Ann Chir Plast Esthet. $2005 ; 50: 118-26$. 